\title{
Short communication: Use of single nucleotide polymorphism genotypes and health history to predict future phenotypes for milk production, dry matter intake, body weight, and residual feed intake in dairy cattle
}

\author{
C. Yao, ${ }^{* 1}$ L. E. Armentano, ${ }^{*}$ M. J. VandeHaar, $†$ and K. A. Weigel ${ }^{*}$ \\ *Department of Dairy Science, University of Wisconsin, Madison 53706 \\ †Department of Animal Sciences, Michigan State University, East Lansing 48824
}

\begin{abstract}
As feed prices have increased, the efficiency of feed utilization in dairy cattle has attracted increasing attention. In this study, we used residual feed intake (RFI) as a measurement of feed efficiency along with its component traits, adjusted milk energy (aMilkE), adjusted dry matter intake (aDMI), and adjusted metabolic body weight (aMBW), where the adjustment was for environmental factors. These traits may also be affected by prior health problems. Therefore, the carryover effects of 3 health traits from the rearing period and 10 health traits from the lactating period (in the same lactation before phenotype measurements) on RFI, aMilkE, aDMI, and aMBW were evaluated. Cows with heavier birth weight and greater body weight at calving of this lactation had significant increases in aMilkE, aDMI, and aMBW. The only trait associated with RFI was the incidence of diarrhea early in the lactation. Mastitis and reproductive problems had negative carryover effects on aMilkE. The aMBW of cows with metabolic disorders early in the lactation was lower than that of unaffected cows. The incidence of respiratory disease during lactating period was associated with greater aMBW and higher aDMI. To examine the contribution of health traits to the accuracy of predicted phenotype, genomic predictions were computed with or without information regarding 13 health trait phenotypes using random forests (RF) and support vector machine algorithms. Adding health trait phenotypes increased prediction accuracies slightly, except for prediction of RFI using RF. In general, the accuracies were greater for support vector machine than RF, especially for RFI. The methods described herein can be used to predict future phenotypes for dairy replacement heifers, thereby facilitating culling decisions that can lead to decreased feed costs during the rearing period. For these decisions, prediction of
\end{abstract}

Received August 4, 2014.

Accepted November 9, 2014.

${ }^{1}$ Corresponding author: chen.yao225@gmail.com the animal's own phenotype is of greater importance than prediction of the genetic superiority or inferiority that will transmit to its offspring.

Key words: health trait, genome-health prediction, feed efficiency

\section{Short Communication}

Feed efficiency is becoming one of the most important topics in animal husbandry because of increasing feed costs. We used residual feed intake (RFI) to measure feed efficiency of dairy cattle, which is the deviation of an animal's feed intake from the expected intake based on production and maintenance within the cohort group (Koch et al., 1963). This definition indicates that differences in RFI of dairy cattle may come from differences between animals in the relationships between feed intake, milk production, and maintenance. Therefore, our study considered RFI and its component traits to gain a better understanding of the causes of variation in feed efficiency.

Feed efficiency and its component traits can be affected by the animal's genetic predisposition, as well as many environmental factors, including the animal's health history. Clinical mastitis has been reported to depress the milk production and may also affect the milk composition (Seegers et al., 2003). Both Urton et al. (2005) and Huzzey et al. (2007) reported cows that later developed metritis consumed less feed than did nonmetritic cows. Subsequent milk production suppression was also reported due to retained placenta, displaced abomasum, milk fever, and ketosis (Deluyker et al., 1991; Rajala-Schultz et al., 1999). However, the carryover effects of these traits on RFI remain unknown. Additionally, the average mature body size of Holstein cattle has been increasing over time in the United States (Hansen, 2000), but the effect of body size on feed efficiency is unclear. Evaluating the effects of these health traits, including body size, may help improve feed efficiency from a management perspective.

The random forests (RF; Breiman, 2001) and support vector machine (SVM; Drucker et al. 1997) are 
2 popular algorithms for prediction. Yao et al. (2013) identified many informative potential epistatic interactions and main effects of SNP on RFI in dairy cattle using RF. Support vector machine was also reported to have the ability to identify and characterize gene-gene and gene-environment interactions (Chen et al., 2008). To further use possible gene-by-gene and gene-by-environment interactions relative to our previous work (Yao et al., 2013), we explored whether adding the health history of animal in the genomic prediction model can improve the accuracy of predicting future phenotypes using RF and SVM. The objectives of the current study were (1) to evaluate carryover effects of 13 health traits on future phenotypes for feed efficiency, milk production, feed intake, and BW, and (2) to explore potential ways to enhance prediction accuracy by using health trait phenotypes and SNP genotypes jointly in the prediction model.

Feed intake and production data were measured from 50 to 200 DIM on 792 lactating Holstein and crossbred cows in the University of Wisconsin-Madison herd. Animal use procedures were approved by the Animal Care and Use Committee of the College of Agricultural and Life Sciences at the University of Wisconsin-Madison. These animals were originally studied in 20 nutrition experiments; more details about those experiments can be found in Ferraretto et al. (2011), Ferraretto et al. (2012), He et al. (2012), Akins et al. (2013), and Akins and Shaver (2014). To be included in the present study, cows were required to have records on daily DMI, daily milk yield, weekly milk composition (fat \%, protein $\%$, and lactose \%), and weekly BW during the study period. For Latin Square experiments, cows were required to complete all diets offered in the experimental design. The expected ranges of milk yield, milk composition, DMI, and BW were set as the population means \pm 5 SD. Records beyond this range were trimmed to the boundary values. One lactation per animal was considered. When data from multiple lactations were available, the lactation with the greatest number of daily observations available was used. Weekly means were calculated for milk yield, milk composition, DMI, and BW. Missing weekly average milk composition and BW were set to the mean values of the previous and following weeks.

According to NRC (2001), the daily milk energy (MilkE) was calculated as

$$
\begin{array}{r}
\text { MilkE }(\text { Mcal })=(0.0929 \times \text { fat } \%+0.0563 \\
\times \text { protein } \%+0.0395 \times \text { lactose } \%) \times \text { DMI }(\mathrm{kg}) .
\end{array}
$$

The weekly mean RFI values of 792 cows were the residual terms calculated as

$$
\begin{gathered}
\mathbf{y = \mu}+\text { YSC }+ \text { ParAge }+ \text { DIM }+ \text { NE }_{\mathrm{L}} \\
+ \text { MBW }+ \text { ration }+ \text { RFI },
\end{gathered}
$$

where $\mathbf{y}$ was a vector of DMI; $\mu$ was the population mean; YSC was the fixed effect of year-by-season at calving with 19 levels (year: 2007 to 2013; season: January to March, April to June, July to September, or October to December); ParAge was the fixed effect of parity-by-age at calving interaction with 20 levels (first parity: $\leq 23,24,25,26$, or $\geq 27$ mo; second parity: $\leq 35$, $36, \ldots, 40$, or $\geq 41 \mathrm{mo}$; third parity and later: $\leq 48$, $49,50,51,52$ to 56,57 to 61,62 to 69 , or $\geq 70 \mathrm{mo}$ ); DIM was the midpoint of DIM during the week from 50 to 200; MBW was the metabolic $\mathrm{BW}$ (i.e., $\mathrm{BW}^{0.75}$ ); ration $\sim N\left(0, \mathbf{I} \sigma_{r}^{2}\right)$ was the random cohort effect, where $\mathbf{I}$ was the identity matrix and $\sigma_{r}^{2}$ was the ration variance; and RFI $\sim N\left(0, \mathbf{I} \sigma_{e}^{2}\right)$ was the random residual, where $\sigma_{e}^{2}$ was the residual variance. Then, the RFI of each animal equaled the mean of all weekly RFI estimates.

Besides RFI, 3 additional traits, adjusted MilkE (aMilkE), adjusted DMI (aDMI), and adjusted MBW (aMBW), were defined for each of 792 animals using linear mixed models:

$$
\begin{aligned}
& \text { MilkE }=\mu_{1}+\text { YSC }+ \text { ParAge }+ \text { DIM }+ \text { ration }+\mathrm{e}_{1}, \\
& \text { DMI }=\mu_{2}+\text { YSC }+ \text { ParAge }+ \text { DIM } \\
& + \text { ration }+\mathrm{e}_{2} \text {, and } \\
& \mathbf{M B W}=\mu_{3}+\text { YSC }+ \text { ParAge }+ \text { DIM } \\
& + \text { ration }+\mathrm{e}_{3} \text {, }
\end{aligned}
$$

where MilkE, DMI, and MBW were vectors of weekly means; $\mu_{1}, \mu_{2}$, and $\mu_{3}$ were population means; YSC, ParAge, DIM, and ration were defined similarly as in model [2]; $\mathrm{e}_{1} \sim N\left(0, \mathbf{I} \sigma_{e_{1}}^{2}\right), \mathrm{e}_{2} \sim N\left(0, \mathbf{I} \sigma_{e_{2}}^{2}\right)$, and $\mathrm{e}_{3} \sim N\left(0, \mathbf{I} \sigma_{e_{3}}^{2}\right)$ were random residuals. Then traits were calculated as adjusted $\mathrm{NE}_{\mathrm{L}}\left(\mathbf{a N E}_{\mathbf{L}}\right)=\hat{\mu}_{1}+\hat{\mathrm{e}}_{1}$, $\operatorname{aDMI}=\hat{\mu}_{2}+\hat{\mathrm{e}}_{2}$, and , and similar to RFI, the aMilkE, aDMI, and aMBW of each cow equaled the average of all her weekly estimates.

A total of 13 health traits were available of 613 cows (179 out of 792 cows with health records unknown) from rearing period (from birth to first calving) and lactating period (from calving to $50 \mathrm{dim}$ in the "current" lactation in which subsequent phenotypes were measured). Traits from the rearing period included birth weight, incidence of scours (SCO), and incidence 
of respiratory disease (RES). From the lactating period, traits included $\mathrm{BW}$ at calving $(\mathbf{c B W})$, BCS at calving (cBCS), calving ease score (CE), incidence of twinning (TWIN), clinical mastitis (MAST), locomotive problems (LOC), metabolic disorders (MET), reproductive problems (REP), diarrhea (DIAR), and respiratory disease $\left(\mathbf{R E S}_{\text {lac }}\right)$. Locomotive problems included feet problems and body injuries. Reproductive disorders included metritis and retained placenta. Ketosis, milk fever, and displaced abomasum were grouped together as metabolic disorders.

The CE scores represented a 5-point scale (no assistance needed, easy pull, hard pull, mechanical pull, and veterinary assistance or $\mathrm{C}$-section). For computation purposes, the first 2 categories were combined as 0 , and the rest were grouped as 1 . The other qualitative traits (SCO, RES, TWIN, MAST, LOC, MET, REP, DIAR, and $\mathrm{RES}_{\text {lac }}$ ) were coded as 0 (unaffected) or 1 (affected at least once). Body condition score was on a 5-point scale with 0.25 intervals (Elanco Animal Health, 1996). The mean values or incidence rates of 13 health traits in our population are shown in Supplementary Table S1 (http://dx.doi.org/10.3168/jds.2014-8707).

The effects of the 13 health traits on RFI, aMilkE, aDMI, and aMBW were evaluated using the linear model as follows:

$$
\begin{gathered}
\mathbf{y}=\mu+\text { birth weight }+\mathrm{SCO}+\mathrm{RES}+\mathrm{cBW} \\
+\mathrm{cBCS}+\mathrm{CE}+\mathrm{TWIN}+\mathrm{MAST}+\mathrm{LOC} \\
+\mathrm{MET}+\mathrm{REP}+\mathrm{DIAR}+\mathrm{RES}_{\mathrm{lac}}+\mathrm{e}
\end{gathered}
$$

where $\mathbf{y}$ was a vector of RFI, aMilkE, aDMI, or aMBW; $\mu$ was the population mean; and $\mathrm{e} \sim N\left(0, \mathbf{I} \sigma_{e}^{2}\right)$ was the random error.

The mixed model equation was solved with the restricted maximum likelihood (REML) method using the R-package "lme4," Version 0.999999-0 (http:// cran.r-project.org/web/packages/lme4/index.html).

Genotypes were obtained from the USDA Agricultural Research Service Animal Genomics and Improvement Programs Laboratory (Beltsville, MD). Missing SNP genotypes were imputed with rounded allele frequencies in the current US Holstein population as of January 2014. The SNP genotypes at each locus were coded as 0,1 , or 2 , counting the number of minor allele copies. The SNP with minor allele frequencies less than $5 \%$ were removed. A total of 57,541 SNP on 465 cows (out of 613 cows) were available for genomic prediction analysis.

Genomic prediction models used in our study included the RF and SVM algorithms. The RF was conducted using the "randomForest" package Version 4.6-6 in R
(Liaw and Wiener, 2011) with $m_{t r y}=1,000$ (the number of features available in each node) and $n_{\text {tree }}=700$ (the number of trees grown) based on results from Yao et al. (2013). To be more stable, each prediction using RF was repeated 100 times. The "svm" function from "e1071" package Version 1.6-1 in R (http://cran.rproject.org/web/packages/e1071/index.html) was used for SVM with default parameters and the radial basis kernel tuned within the training set.

The 465 cows with genotypes were assigned to the training and testing sets based on birthdate. The training set comprised 384 cows with birthdate before January 1,2010 , whereas the testing set comprised 78 cows born after January 1, 2010. The genomic predictions were performed with (1) SNP genotypes and (2) SNP genotypes plus 13 health trait phenotypes. Accuracies were measured in the testing set as correlations between phenotypes (RFI, aMilkE, aDMI, and aMBW) and their predicted values from RF and SVM, which were trained in the training set.

Residual feed intake, aMilkE, aDMI, and aMBW were calculated for 792 cows, and their summary statistics, including mean, $\mathrm{SD}$, minimum, and maximum, are presented in Supplementary Table S2 (http://dx.doi. org/10.3168/jds.2014-8707). The reference levels for ParAge and YSC in both models [2] and [3] were second parity and $36 \mathrm{mo}$ of age at calving and calving between October and December 2010. The DIM was fitted as the deviation from the average dim in the population (134 DIM) for each record. Thus, the interpretation of aMilkE (similarly for aDMI and aMBW) was an equivalent daily MilkE produced by the cow, standardized to about 134 DIM in the second parity calved at 36 mo of age between October and December 2010.

Effects of health data on RFI, aMilkE, aDMI, and aMBW were evaluated using a linear model. Their estimated coefficients and significant levels are listed in Table 1. Heavier birth weight and cBW (within the parity) phenotypes were significantly associated with increased aMilkE, aDMI, and aMBW. Birth weight had a larger estimate than $\mathrm{cBW}$, which may be because the $\mathrm{SD}$ of $\mathrm{cBW}$ is much greater than the SD of birth weight. Additionally, though mature weight is moderated correlated with birth weight (Bullock et al., 1993; Northcutt and Wilson, 1993), cBW can be affected by more environmental factors than birth weight, and this may dilute the apparent effect of $\mathrm{cBW}$.

Though their coefficients were positive, neither birth weight nor cBW affected RFI, which suggests that cows with smaller birth weight and cBW tend to be slightly more efficient. In our study, RFI was phenotypically independent of birth weight and cBW, with correlations of 0.07 and 0.004 , respectively. Whether selection for improved RFI in dairy cattle will result in a significant 
Table 1. Estimated effects of health traits on residual feed intake (RFI), adjusted net energy for milk (aMilkE), adjusted dry matter intake (aDMI), and adjusted metabolic body weight (aMBW) for 613 cows in the present study

\begin{tabular}{|c|c|c|c|c|}
\hline Health $^{1}$ & $\begin{array}{c}\mathrm{RFI} \\
(\mathrm{kg} / \mathrm{d})\end{array}$ & $\begin{array}{c}\text { aMilkE } \\
\text { (Mcal/d) }\end{array}$ & $\begin{array}{l}\mathrm{aDMI} \\
(\mathrm{kg} / \mathrm{d})\end{array}$ & $\begin{array}{c}\underset{\left(\mathrm{kg}^{0.75}\right)}{\operatorname{aMBW}} \\
\end{array}$ \\
\hline Intercept $^{2}$ & -0.115 & $32.117^{* * *}$ & $26.447^{* * *}$ & $126.891^{* * *}$ \\
\hline \multicolumn{5}{|l|}{ Rearing period } \\
\hline Birth weight & 0.023 & $0.058^{*}$ & $0.067^{* * *}$ & $0.230^{* * *}$ \\
\hline Scour & 0.214 & 0.014 & 0.232 & 0.159 \\
\hline RES & 0.054 & 0.390 & 0.125 & -0.695 \\
\hline \multicolumn{5}{|l|}{ Lactating period } \\
\hline $\mathrm{cBW}$ & 0.000 & $0.005^{* *}$ & $0.005^{* * *}$ & $0.028 * * *$ \\
\hline $\mathrm{cBCS}$ & -0.003 & 0.657 & 0.293 & 0.579 \\
\hline $\mathrm{CE}$ & 0.193 & -0.015 & 0.171 & -0.116 \\
\hline TWIN & -0.130 & 0.403 & -0.007 & -0.374 \\
\hline $\mathrm{LOC}^{3}$ & 0.261 & -0.529 & 0.019 & -0.490 \\
\hline MAST & -0.115 & $-1.196^{*}$ & -0.449 & 0.860 \\
\hline $\mathrm{MET}^{4}$ & -0.120 & 0.290 & -0.226 & $-1.936^{* *}$ \\
\hline $\mathrm{REP}^{5}$ & 0.086 & $-1.060^{*}$ & -0.273 & 0.155 \\
\hline $\mathrm{RES}_{\mathrm{lac}}$ & 0.371 & 0.360 & $0.744^{*}$ & $2.257^{*}$ \\
\hline DIAR & $1.988^{* *}$ & -1.585 & 1.042 & -3.531 \\
\hline
\end{tabular}

${ }^{1}$ DIAR $=$ diarrhea (during lactating period), RES $=$ respiratory disease, $\mathrm{RES}_{(\mathrm{lac})}=\mathrm{RES}$ during lactating period, $\mathrm{cBW}=$ calving body weight, $\mathrm{cBCS}=$ calving $\mathrm{BCS}, \mathrm{CE}=$ calving ease, TWIN $=$ twining, MAST $=$ mastitis, $\mathrm{LOC}=$ locomotive problems, $\mathrm{MET}=$ metabolic disorders, $\mathrm{REP}=$ reproductive problems.

${ }^{2}$ Intercept represented the mean value of animals with birth weight $=40 \mathrm{~kg}$, no scour, no RES, $\mathrm{cBW}=667$ $\mathrm{kg}, \mathrm{cBCS}=3.5, \mathrm{CE}=0$, no TWIN, no MAST, no MET, no LOC, no Repro, no RES lac, and no scour during lactation.

${ }^{3}$ Locomotive problems included feet problems and body injury.

${ }^{4}$ Metabolic disorders included ketosis, milk fever, and displaced abomasum.

${ }^{5}$ Reproductive problems included metritis and retained placenta.

${ }^{*} P<0.05,{ }^{* *} P<0.01,{ }^{* * *} P<0.001$.

change in body size depends on the definition of RFI, and further studies regarding the optimal definition are needed. The lack of association between RFI and cBCS was interesting, because cows with higher cBCS scores were not more efficient. The reason may be that slightly higher milk production for cows with greater cBCS were accompanied by small increases in feed intake, and net efficiency was not improved. The RFI calculated in our study was also a measure of efficiency that forgave large cows for their large body sizes, and net efficiency was not harmed either. This suggests that selection for larger cows may increase milk production and growth rate, but also increased maintenance requirements and therefore no improvement in feed efficiency.

The only trait significantly associated with RFI was the incidence of diarrhea during the lactating period before phenotype measurements $(P<0.01)$, though the incidence rate was only $1 \%$ in our population. Instances of diarrhea may have been missed if they were not observed during feeding and milking time. The mean RFI of affected cows was greater (less efficient) than unaffected cows. Effects of diarrhea on other traits were slightly increased aDMI, slightly decreased aMilkE, and slightly lower aMBW, but none of these associations were significant. The incidence of scours during the rearing period also appeared to have a small negative effect on RFI, but was not significant $(P=0.16)$. Eliminating the incidence of scours may improve the feed efficiency of cows, but the effects and mechanism of scours during the rearing and lactating periods should be studied further.

Regarding milk production, mastitis and reproductive problems had significant negative carryover effects $(P<0.05)$. Mastitis was reported to have slightly unfavorable genetic correlation with milk production (Emanuelson et al., 1988), and suppressed milk production was also observed during endotoxin-induced mastitis (Shuster et al., 1991). Multiparous cows affected by metritis, a component trait of our reproductive problems, were also found to produce less milk (Wittrock et al., 2011). Therefore, the negative effects of mastitis and reproductive problems on milk production may not be only during the affection, but also carried over to the later stage of lactation.

Other traits that showed significant effects on aMBW and aDMI included metabolic disorders and the incidence of respiratory disease during lactating period. The aMBW of unaffected cows was higher than that of cows affected by MET $(P<0.01)$, and lower than that of cows affected by $\operatorname{RES}_{\text {lac }}(P<0.05)$. In addition, incidence of $\mathrm{RES}_{\text {lac }}$ was found to be associated with greater aDMI $(P<0.05)$, but the mechanism underly- 
Table 2. Accuracies (\%) of genomic prediction of future phenotypes with or without information regarding health trait phenotypes on residual feed intake (RFI), adjusted net energy for milk (aMilkE), adjusted dry matter intake (aDMI), and adjusted metabolic body weight (aMBW); the accuracy was the correlation between phenotypes and predicted values using random forests (RF) and support vector machine (SVM) algorithms

\begin{tabular}{lcccc}
\hline Item & RFI & aMilkE & aDMI & aMBW \\
\hline RF & & & & \\
SNP genotype & 8.76 & 21.15 & 28.73 & 37.20 \\
SNP genotype + health phenotype & 8.12 & 22.30 & 29.55 & 38.23 \\
SVM & & & & \\
SNP genotype & 20.45 & 32.62 & 35.88 & 34.83 \\
SNP genotype + health phenotype & 20.45 & 32.66 & 35.92 & 34.90 \\
\hline
\end{tabular}

ing increased feed intake and BW in cows previously affected by respiratory disease is unclear.

Genomic predictions with or without information regarding 13 health trait phenotypes were also performed to examine their contributions to predict phenotypes. The accuracies were measured as correlations between phenotypes and their predicted values using $\mathrm{RF}$ and SVM (Table 2). In general, adding health traits increased prediction accuracies slightly, except in prediction of RFI using RF. The improvements tended to be greater when using RF. The prediction accuracies were higher for SVM than RF for 3 of 4 traits, especially for RFI, where the accuracy of SVM was more than twice that of RF.

The RFI, aMilkE, aDMI, and aMBW measurements used in our study were phenotypes, not EBV. They represented combinations of additive, dominance, and epistatic genetic effects and some permanent environmental effects (e.g., health history). By design, RF and SVM are able to use interactive relationship between predictors. Adding health trait phenotypes into the genomic prediction model may capture interactions between genetic markers and environmental factors, and this may contribute to the improvement in predictive performance of RF and SVM observed in our study. However, the small magnitude of improvement possibly reflected a lack of precision in the diagnosis or reporting of health problems, and whole-genome SNP may already contain substantial information about an animal's predisposition for traits such as BW and susceptibility to infectious diseases and metabolic disorders.

Our results showed that greater birth weight and BW at calving were significantly associated with increased aMilkE, aDMI, and aMBW, but little or no change in RFI. Therefore, selection for larger cows may increase milk production, but it may also result in higher maintenance requirements and no improvement in feed efficiency. Reducing the incidence of scours, respiratory disease, mastitis, and reproductive disorders during rearing or lactating periods may help improve feed efficiency and overall productivity and welfare in dairy cattle. In genome-enabled prediction, health history may contribute to the improvement in predictive performance of RF and SVM.

\section{ACKNOWLEDGMENTS}

The assistance of Francisco E. Contreras-Govea and Sandy Bertics, both of the Department of Dairy Science, University of Wisconsin-Madison with data preparation is gratefully noted. This project was supported by Agriculture and Food Research Initiative Competitive Grants no. 2008-35205-18711 and 2011-68004-30340 from the USDA National Institute of Food and Agriculture (Washington, DC). Support from Hatch grant no. MSN139239 from the Wisconsin Agricultural Experiment Station (Madison, WI) is acknowledged, and K. A. Weigel acknowledges partial financial support from the National Association of Animal Breeders (Columbia, MO).

\section{REFERENCES}

Akins, M. S., S. J. Bertics, M. T. Socha, and R. D. Shaver. 2013. Effects of cobalt supplementation and vitamin B12 injections on lactation performance and metabolism of Holstein dairy cows. J. Dairy Sci. 96:1755-1768.

Akins, M. S., and R. D. Shaver. 2014. Effect of corn snaplage on lactation performance by dairy cows. Prof. Anim. Sci. 30:86-92.

Breiman, L. 2001. Random forests. Mach. Learn. 45:5-32.

Bullock, K. D., J. K. Bertrand, and L. L. Benyshek. 1993. Genetic and environmental parameters for mature weight and other growth measures in polled Hereford cattle. J. Anim. Sci. 71:1737-1741.

Chen, S. H., J. Sun, L. Dimitrov, A. R. Turner, T. S. Adams, D. A. Meyers, B. L. Chang, S. L. Zheng, H. Gronberg, J. Xu, and F. C. Hsu. 2008. A support vector machine approach for detecting genegene interaction. Genet. Epidemiol. 32:152-167.

Deluyker, H. A., J. M. Gay, L. D. Weaver, and A. S. Azari. 1991. Change in milk yield with clinical diseases for a high producing dairy herd. J. Dairy Sci. 74:436-445.

Drucker, H., C. J. C. Burges, L. Kaufman, A. Smola, and V. Vapnik. 1997. Support vector regression machines. Adv. Neural Inf. Process. Syst. 9:155-161.

Elanco Animal Health. 1996. Body Condition Scoring in Dairy Cattle. A1:8478. Elanco Animal Health, Greenfield, IN.

Emanuelson, U., B. Danell, and J. Philipsson. 1988. Genetic parameters for clinical mastitis, somatic cell counts, and milk production by multiple-trait restricted maximum likelihood. J. Dairy Sci. $71: 467-476$.

Ferraretto, L. F., R. D. Shaver, and S. J. Bertics. 2012. Effect of dietary supplementation with live-cell yeast at two dosages on lacta- 
tion performance, ruminal fermentation, and total-tract nutrient digestibility in dairy cows. J. Dairy Sci. 95:4017-4028.

Ferraretto, L. F., R. D. Shaver, M. Espineira, H. Gencoglu, and S. J. Bertics. 2011. Influence of a reduced-starch diet with or without exogenous amylase on lactation performance by dairy cows. J. Dairy Sci. 94:1490-1499.

Hansen, L. B. 2000. Consequences of selection for milk yield from a geneticist's viewpoint. J. Dairy Sci. 83:1145-1150.

He, M., K. L. Perfield, H. B. Green, and L. E. Armentano. 2012. Effect of dietary fat blend enriched in oleic or linoleic acid and monensin supplementation on dairy cattle performance, milk fatty acid profiles, and milk fat depression. J. Dairy Sci. 95:1447-1461.

Huzzey, J. M., D. M. Veira, D. M. Weary, and M. A. G. von Keyserlingk. 2007. Prepartum behaviour and dry matter intake identify dairy cows at risk for metritis. J. Dairy Sci. 90:3220-3233.

Koch, R. M., L. A. Swiger, D. Chambers, and K. E. Gregory. 1963. Efficiency of feed use in beef cattle. J. Anim. Sci. 22:486-494.

Liaw, A., and M. Wiener. 2011. randomForest: Breiman and Cutler's Random Forests for classification and regression. R Package, ver. 4.6-3.

NRC. 2001. Nutrient Requirements of Dairy Cattle. 7th rev. ed. Natl. Acad. Sci., Washington, DC.

Northcutt, S. L., and D. E. Wilson. 1993. Genetic parameter estimates and expected progeny differences for mature size in Angus cattle. J. Anim. Sci. 71:1148-1153.
Rajala-Schultz, P. J., Y. T. Grohn, and C. E. McCulloch. 1999. Effects of milk fever, ketosis, and lameness on milk yield in dairy cows. J. Dairy Sci. 82:288-294.

Seegers, H., C. Fourichon, and F. Beaudeau. 2003. Production effects related to mastitis and mastitis economics in dairy cattle herds. Vet. Res. 34:475-491.

Shuster, D. E., R. J. Harmon, J. A. Jackson, and R. W. Hemken. 1991. Suppression of milk production during endotoxin-induced mastitis. J. Dairy Sci. 74:3763-3774

Urton, G., M. A. G. von Keyserlingk, and D. M. Weary. 2005. Feeding behavior identifies dairy cows at risk for metritis. J. Dairy Sci. $88: 2843-2849$

Wittrock, J. M., K. L. Proudfoot, D. M. Weary, and M. A. G. von Keyserlingk. 2011. Short communication: Metritis affects milk production and cull rate of Holstein multiparous and primiparous dairy cows differently. J. Dairy Sci. 94:2408-2412.

Yao, C., D. M. Spurlock, K. A. Weigel, L. E. Armentano, C. D. Page Jr., and M. J. VandeHaar. 2013. Random Forests approach for identifying additive and epistatic single nucleotide polymorphisms associated with residual feed intake in dairy cattle. J. Dairy Sci. 96:6716-6729. 